\title{
Characterising the Effects of Strain Rate, Crystallographic Texture and Direction of Loading on the Mechanical Behaviour of Ti-6Al-4V
}

\author{
E. Wielewski ${ }^{1,2} \cdot$ M. R. Arthington ${ }^{2}$ C. R. Siviour ${ }^{2} \cdot$ N. Petrinic ${ }^{2}$
}

Received: 2 October 2015/ Accepted: 30 October 2015/Published online: 3 November 2015

(C) Society for Experimental Mechanics, Inc 2015

\begin{abstract}
A cross-rolled plate of the industrially important titanium alloy, Ti-6Al-4V, has been microstructurally and mechanically characterised using a range of different experimental techniques. The microstructure of the material has been studied using backscatter electron (BSE) microscopy and electron backscatter diffraction (EBSD), with the crystallographic orientation data from the EBSD used to reconstruct the orientation distribution function of the dominant $\alpha$ phase. The mechanical behaviour of the material has been investigated at quasi-static and high strain rates in the three orthogonal material orientations in both tension and compression. A novel in situ optical measurement technique has been used to measure the geometry of the specimens during both quasi-static and high strain rate mechanical testing, improving the accuracy of the mechanical testing results and providing unprecedented information about the evolving geometries of the specimens. The macroscopic stress-strain response and the evolution of specimen cross-sectional profiles have been qualitatively linked to the macroscopic crystallographic texture in the plate.
\end{abstract}

Keywords Titanium $\cdot$ Mechanical properties $\cdot$ High strain rate Electron backscatter diffraction - Texture

C. R. Siviour

clive.siviour@eng.ox.ac.uk

E. Wielewski

euan.wielewski@glasgow.ac.uk

1 School of Engineering, University of Glasgow, Glasgow G12 8QQ, UK

2 Department of Engineering Science, University of Oxford, Oxford OX1 3PJ, UK

\section{Introduction}

Titanium alloys have seen widespread use in the aerospace and defence industries due to their high specific strength and good resistance to corrosion [1-3]. By far the most widely used of the titanium alloys is Ti-6Al-4V, owing to its excellent balance of strength, ductility and manufacturability [2]. Despite these favourable properties, high production costs have limited the use of titanium alloys to relatively niche applications where weight is a critical design factor. However, new production methods and lowcost variants of traditional titanium alloys have the potential to bring production costs down to a level where titanium alloys will become attractive structural materials for the automotive industry [4].

If titanium alloys are to be exploited more widely it is vital that their mechanical behaviour is better understood, particularly in terms of their in-service properties and behaviour during key manufacturing processes, such as sheet forming. This requires the development of novel mechanical characterisation methods, capable of capturing the complex behaviour of these materials, coupled with extensive microstructural characterisation, such that microstructural and crystallographic features can be explicitly linked to mechanical behaviour.

The wide range of microstructures that can develop in titanium alloys during thermo-mechanical processing complicates this later point significantly. Titanium is an allotropic material, with a high temperature body-centred cubic (bcc) phase, known as the $\beta$ phase, and a low temperature hexagonal close-packed (hcp) phase, known as the $\alpha$ phase. In Ti-6Al-4V the alloying elements aluminium and vanadium are included as $\alpha$ and $\beta$ phase stabilisers, respectively. This alloying results in a small volume fraction of the $\beta$ phase being retained at room temperature 
(typically 5-10\%). Such alloys are commonly referred to as $\alpha+\beta$ alloys, with Ti-6Al-4V being an example of a "classic" $\alpha+\beta$ alloy [1-3]. On cooling from the $\beta$ or $\alpha+\beta$ phase field, secondary (or transformed) $\alpha$ is produced, which can form as either grains or platelets. The orientation of the transformed $\alpha$ is linked to the prior $\beta$ through the Burgers orientation relation [5]:

$$
\begin{gathered}
\{110\}_{\beta} \|(0002)_{\alpha} \\
{[111]_{\beta} \|[11 \overline{2} 0]_{\alpha}}
\end{gathered}
$$

As a result of this orientation relation and restricted crystallographic slip during thermo-mechanical processing, complex microstructures can be developed with non-random crystallographic orientation distributions at the micro and macro scales. To better understand the link between these complex microstructures and the mechanical behaviour of titanium alloys, we must first consider the fundamental nature of elastic and plastic deformation in Ti-6Al$4 \mathrm{~V}$.

Due to the low volume fraction $(\sim 5-10 \%)$ of the $\beta$ phase typically present in $\mathrm{Ti}-6 \mathrm{Al}-4 \mathrm{~V}$, it is generally assumed that the bcc $\beta$ phase plays a secondary role in the bulk mechanical behaviour of Ti-6Al-4V. As a result, this work will focus on the dominant hcp $\alpha$ phase.

The nature of elastic deformation of the hexagonal unit cell gives rise to a number of interesting points. Firstly, due to the fact that hcp single crystals are elastically transversely anisotropic, the elastic response can vary significantly depending on the orientation of a given crystal relative to the imposed loading conditions. For example, the Young's modulus (E) of a titanium single crystal varies between 100-145 GPa depending on the declination angle of the c-axis relative to an applied uniaxial stress [1]. Secondly, unlike cubic systems, dilatational strains result in deviatoric stresses. This means that the deviatoric stresses (which drive plastic deformation) cannot be decoupled from the hydrostatic stresses.

Metals plastically deform principally by the glide of dislocations along prefered planes in the close-packed lattice directions. In the hcp $\alpha$ phase of titanium, the easy glide of dislocations occurs in the $\langle 11 \overline{2} 0\rangle$ directions along the $\{10 \overline{1} 0\},(0001)$ and $\{10 \overline{1} 1\}$ planes [6]. These are also known as the prismatic, basal and pyramidal planes, respectively. Due to the common $\langle 11 \overline{2} 0\rangle$ slip direction, these slip systems are collectively referred to as $\langle a\rangle$ type slip systems (Fig. 1).

At low strain rates non- $\langle a\rangle$ type plastic deformation can be accommodated by so-called $\langle a+c\rangle$ type slip on the pyramidal planes (Fig. 1). Conversely, at high strain rates, it has been shown that deformation twinning plays an important role in accommodating deformation in non- $\langle a\rangle$

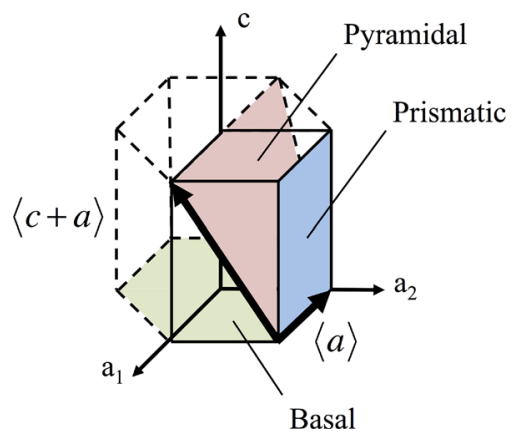

Fig. 1 Typical active slip systems in hcp $\alpha$ phase of titanium

type directions [7-11], although it should be noted that there is evidence of twinning playing a role at quasi-static strain rates too [12]. It is generally accepted that $\langle c+a\rangle$ type slip systems have a higher critically resolved shear stress (CRSS) than the $\langle a\rangle$ type slip systems, leading to significant anisotropic behaviour at the single crystal level.

The interaction of these anisotropic elastic and plastic deformation mechanisms at the microstructural level can result in highly complex local mechanical behaviour [1315]. However, for the case of bulk deformation at the macroscopic scale, behaviour is generally driven by the preferred crystallographic orientation, also known as macrotexture or simply texture.

Due to the industrial importance of Ti- $6 \mathrm{Al}-4 \mathrm{~V}$, its mechanical response has been extensively studied under a wide range of mechanical loading conditions. Despite this, limited data is available in the literature on the high strain rate mechanical behaviour of Ti-6Al-4V, with data particularly limited for loading in different material orientations and directions (tension/compression). This is compounded by the fact that compositional, microstructural and/or crystallographic texture data are often not included, making it difficult to meaningfully compare results.

In this paper, the bulk mechanical behaviour of an industrially important Ti-6Al-4V plate material has been investigated at quasi-static and high strain rates, with a novel in situ optical measurement technique used to precisely measure the evolving cross-sectional area of the specimens during experiments. Mechanical testing was performed under both uniaxial tension and compression in the three orthogonal material directions, relative to the processing of the plate. The as-received material has been microstructurally and crystallographically characterised using backscatter electron (BSE) microscopy and electron backscatter diffraction (EBSD). The macroscopic stressstrain response and the evolution of specimen cross-sectional profiles have then been qualitatively linked to the macroscopic crystallographic texture in the plate. 


\section{Material}

\section{General}

The material investigated in this paper was a cross-rolled Ti-6Al-4V plate, typical of that used in many industrial applications. The plate was from the traditional triple vacuum arc remelt route, with secondary processing consisting of cross-rolling in the $\alpha+\beta$ phase field. The received plate was $20 \mathrm{~mm}$ thick and was designated the material orientations; rolling direction 1 (RD1), rolling direction 2 (RD2) and through-thickness direction (TT), as shown in Fig. 2.

\section{Microstructure}

To characterize the microstructure of the as-received plate, BSE microscopy was carried out normal to the TT direction of the plate. The material was prepared for microscopy by multi-stage coarse grinding using $\mathrm{SiC}$ paper and diamond paste, followed by a chemical/mechanical polish using a neoprene pad and colloidal silica solution. The BSE microscopy in this study was carried out using a FEI Quanta $^{\mathrm{TM}} 250$ scanning electron microscope. A representative BSE image of the Ti-6Al-4V plate is given in Fig. 3.

As can be seen from Fig. 3, the as-received Ti-6Al-4V plate has a typical equiaxed microstructure consisting of $\alpha$ grains (dark regions) and a small volume fraction of retained $\beta$ (light regions) at the interfaces between $\alpha$ grains.

\section{Texture}

EBSD analysis was carried out to characterize the crystallographic texture of the dominant $\alpha$ phase of the Ti-6Al$4 \mathrm{~V}$ plate. As with the BSE microscopy, all data were

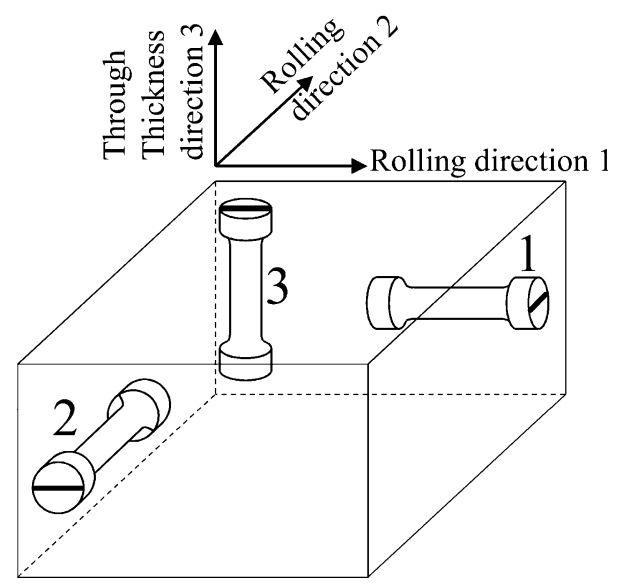

Fig. 2 A schematic diagram of the material and specimen orientations from the parent plate

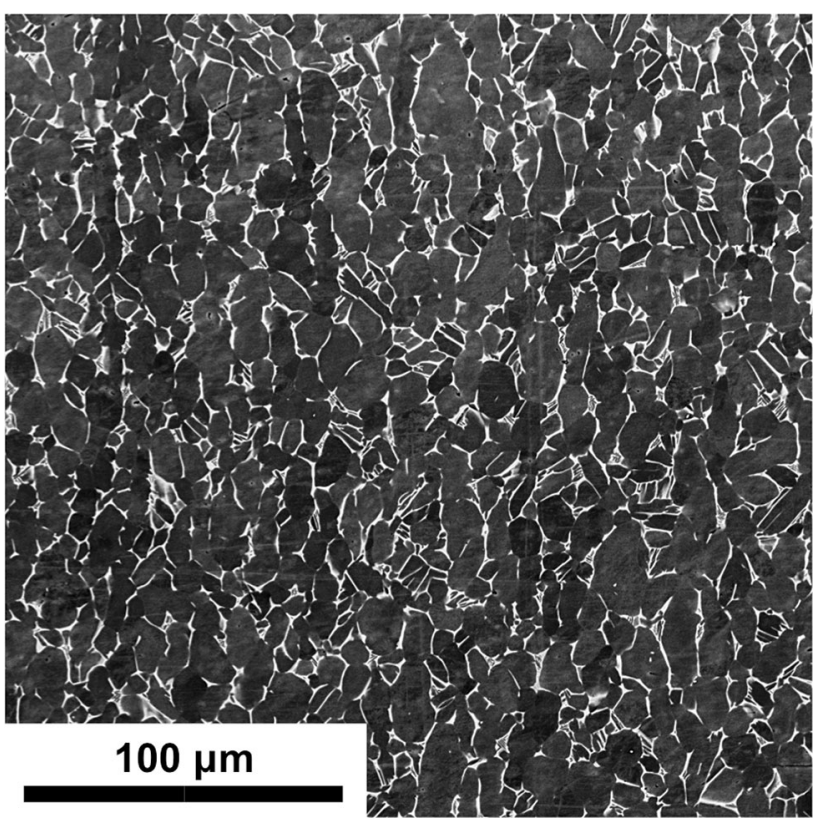

Fig. 3 BSE image of CR-Ti-6Al-4V plate microstructure from the $\mathrm{RD} 1$ (vertical direction)/RD2 (horizontal direction) plane

normal to the TT direction. The EBSD analyses in this study were carried out using a Carl Zeiss Evo LS15 variable pressure scanning electron microscope equipped with a $\mathrm{LaB}_{6}$ electron gun and an EDAX OIM XM 4 Hikari System equipped with a DigiView IV Camera.

To characterize the macroscopic texture of the plate, a large area EBSD scan was completed over an area of $\sim 2 \times 2 \mathrm{~mm}$ with a step size of $10 \mu \mathrm{m}$. The orientation distribution function (ODF) of the hcp $\alpha$ phase was determined from the EBSD data using a finite element representation of the hexagonal fundamental region of Rodrigues space [16] with a total of 6010 independent nodal points and 36,000 elements, as shown in Fig. 4a. The ODF is shown as a multiple of a uniform distribution (MUD) plot over the fundamental region in Fig. 4b, with sections through the fundamental region given in Fig. 4c.

The ODF was used to generate pole figures to highlight any crystallographic texturing seen in the plate. Using the unit sphere mesh shown in Fig. 5a, pole figures for the (0001) and $\{10 \overline{10}\}$ planes are given as MUD plots in Fig. 5b. From these plots it can be seen that the $\alpha$ phase exhibits relatively strong texturing, with a significant fraction of grains having the c-axis of the hcp unit cell aligned with the orthogonal rolling directions of the plate. Further, there is a stronger preference for grains to have their c-axis aligned with RD1, than compared to RD2. Some light texturing is observed around the c-axis in these principal directions.

To characterize the microscopic texture of the plate, two smaller area EBSD scans were completed; one over an area 
Fig. 4 a Hexagonal

fundamental region mesh and b the hcp $\alpha$ phase ODF over the hexagonal fundamental region in MUD

Fig. 5 a Unit sphere mesh and b pole figure plots of the hcp $\alpha$ phase from the ODF in MUD (a)

(b)

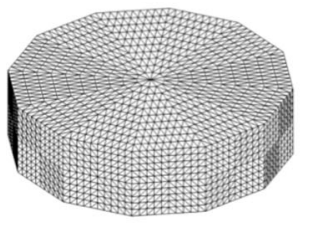

$\max =3.97$
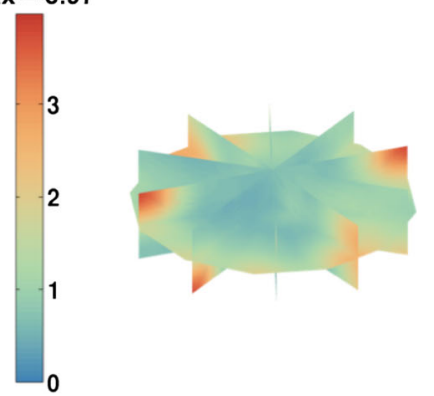

(a)

(b)

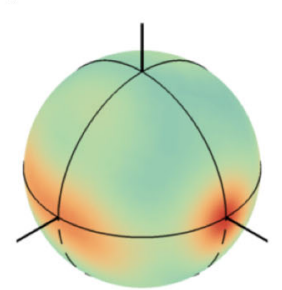

(0001)

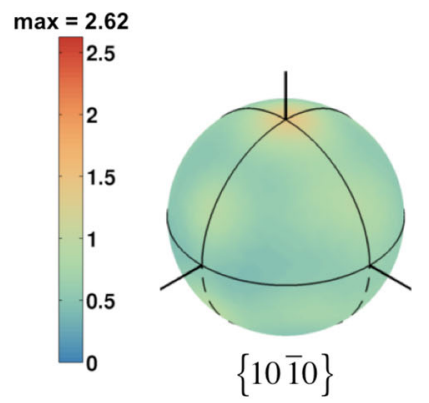

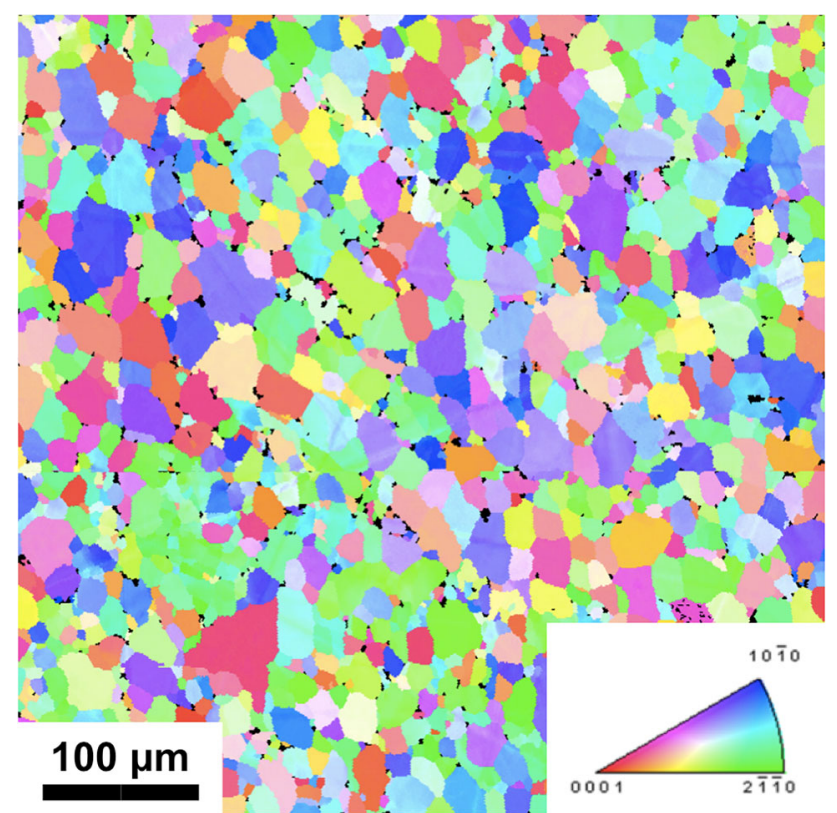

Fig. 6 IPF map of the hcp $\alpha$ phase from RD1 (vertical direction)/ $\mathrm{RD} 2$ (horizontal direction) plane

of $500 \times 500 \mu \mathrm{m}$ with a step size of $1 \mu \mathrm{m}$ and the other over an area of $100 \times 100 \mu \mathrm{m}$ with a step size of $0.1 \mu \mathrm{m}$. The data from these scans were then used to generate inverse pole figure (IPF) maps of the scanned regions, with the results for the larger area scan given in Fig. 6 and the results for the smaller area scan given in Fig. 7.

It is well documented that Ti-6Al-4V from similar processing routes to those of the plate studied here can

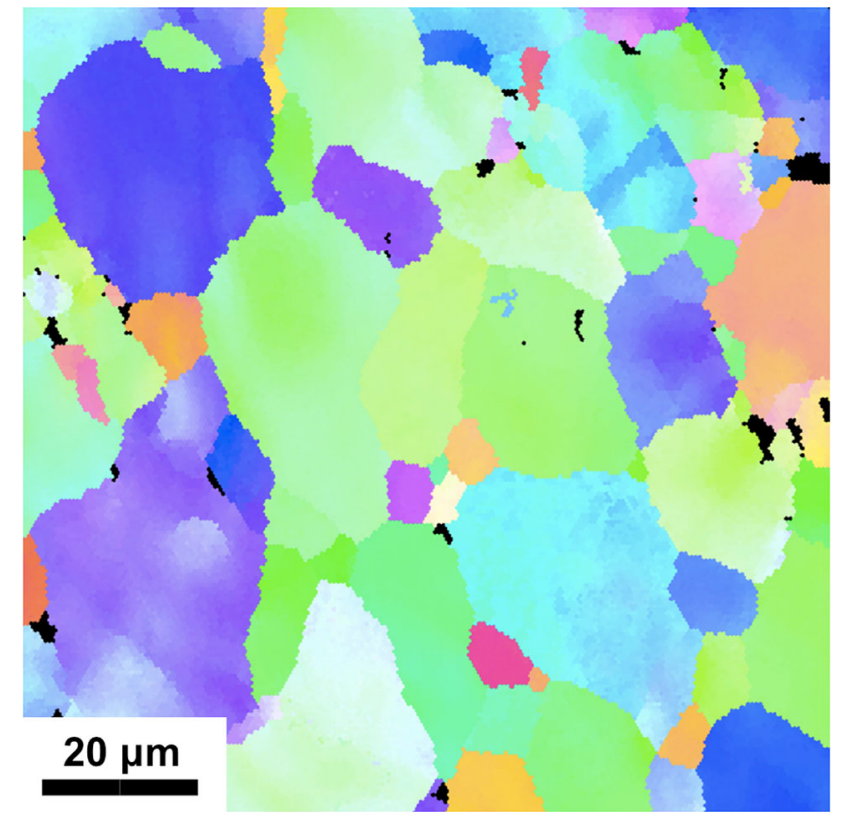

Fig. 7 High resolution IPF map of the hcp $\alpha$ phase from the RD1 (vertical direction)/RD2 (horizontal direction) plane

have large regions where crystals of a similar orientation are clustered together. These regions are commonly referred to as micro-texture or macrozones and can affect mechanical behaviour under conditions where local microstructural features are of importance, such as fatigue loading and spall failure [17-19]. However, the characterisation of macrozone features is of little relevance here 
as the focus of this paper is on bulk deformation behaviour, which is principally dependent on macroscopic crystallographic texturing.

Interestingly, as observed from the higher-resolution EBSD data shown in Fig. 7, there is some intra-granular misorientation present in the $\alpha$ phase of the as-received material. The presence of intra-granular misorientation indicates some level of residual stress, potentially leading to perceived anisotropy and tension/compression asymmetry of yield.

\section{Experiments}

\section{Overview}

Both quasi-static and high strain rate uniaxial tension and compression experiments were performed in the three orthogonal material directions, relative to the processing of the plate. The tensile specimens had the dimensions shown in Fig. 8; these dimensions are a compromise between the requirement for stress equilibrium at high strain rates and the desire for a large length/diameter ratio in uniaxial testing. The compression specimens were right cylinders of nominal dimensions $3.0 \mathrm{~mm}$ diameter by $3.5 \mathrm{~mm}$ length.

The specimens were cut from the cross-rolled Ti-6Al$4 \mathrm{~V}$ plates in the three principal orthogonal material directions, orientated as shown in Fig. 2. The plate material was prepared in two different forms; one was the as-received $20 \mathrm{~mm}$ thick plate, the other a pack of three as-received plates that had been diffusion bonded together to make a $60 \mathrm{~mm}$ thick plate. This provided a thick enough plate to manufacture through-thickness tensile specimens, for which the centre of the $8 \mathrm{~mm}$ gauge sections were positioned at the centre of the middle plate.

Three or more repeats of each experimental condition were performed with a single representative result for each presented here, apart from the high strain rate tension condition, where only one specimen of each was tested.

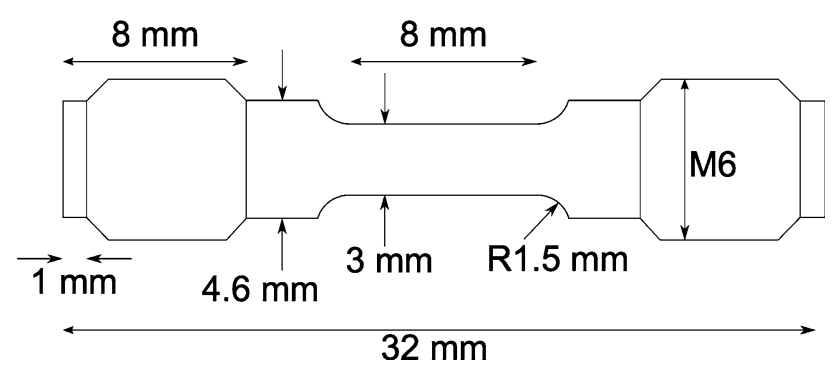

Fig. 8 Tensile specimen design, used for both quasi-static and high strain rate testing

\section{In-Situ Geometry Reconstruction Technique}

Optical reconstruction techniques can be employed in order to accurately measure changes in specimen geometry during uniaxial mechanical experiments. For initially cylindrical specimens of isotropic materials, which retain circular cross-sections throughout a uniaxial mechanical experiment, photographs from a single orthogonal frame are sufficient to fully reconstruct the specimen geometry. However, there are many materials for which this is not the case. As discussed previously, metals such as titanium have mechanical properties that are highly dependent on crystallographic orientation and, if their production route leads to the formation of macrotexture, initially circular crosssections will deform into ellipses during a uniaxial mechanical experiment.

The authors have developed a technique by which the elliptical cross-section of a specimen can be reconstructed from photographs of the specimen from three different perspectives. Outline profiles are determined in each of the photographs using an edge-detection algorithm. These profiles are then used to calculate six lines at regularly spaced axial positions that are tangential to the specimen's surface. Five unique tangential lines are sufficient to describe a general ellipse, and the six lines together are used in a least-squares optimisation to find the ellipse that minimises the distance to the lines at every position.

The reconstruction at distinct axial positions produces the five key parameters that define an ellipse: lengths of semi-major $\left(r_{a}\right)$ and semi-minor $\left(r_{b}\right)$ axes, orientation $(\varphi)$ and the $\mathrm{x}$ and $\mathrm{y}$ coordinates of the centre of the ellipse, these parameters are defined in Fig. 9.

By repeating the photographs throughout the duration of the experiment, a deformation history can be constructed. Recent papers have described and validated the reconstruction process in detail $[20,21]$, and also shown it's utility when applied to Taylor impact experiments on commercially pure zirconium [22] and Ti-6Al-4V [19].

The setup used in these experiments was identical to that described in [20], the only difference being that the illumination set-up was modified such that all pictures of the

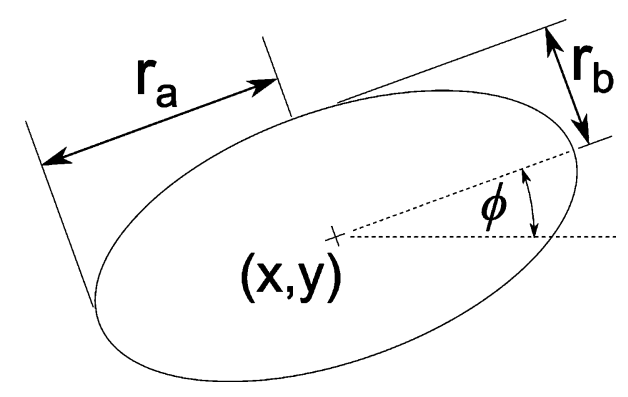

Fig. 9 Definition of ellipse parameters 
specimens were silhouettes, which aided the edge-detection process.

\section{Quasi-Static Testing}

The quasi-static experiments were performed using a commercial screw-driven device. For the tensile experiments, one end of the specimen was displaced at a constant $5.6 \times 10^{-6} \mathrm{~m} \mathrm{~s}^{-1}$, resulting in a nominal strain rate of $7.0 \times 10^{-4} \mathrm{~s}^{-1}$. For the compression experiments, the upper platen was displaced at a constant $4.6 \times 10^{-3} \mathrm{~m} \mathrm{~s}^{-1}$, corresponding to a nominal strain rate of $1.3 \times 10^{-3} \mathrm{~s}^{-1}$.

The optical measurement configuration consisted of the three 4 MP TELI digital cameras fixed at an angular spacings of $45^{\circ}$. Photos were captured simultaneously by all three cameras at a rate of one every $3 \mathrm{~s}$.

Experiments conducted under these conditions will be referred to as quasi-static (QS).

\section{High Strain Rate Testing}

Split-Hopkinson pressure bars were used to characterise the mechanical response of the Ti-6Al-4V plate to high strain rate deformation [23, 24]. The high strain rate tensile experiments were performed using a tensile split-Hopkinson bar $[25,26]$ with $10 \mathrm{~mm}$ diameter input and output rods of Ti-6Al-4V and phosphor bronze, respectively. The high strain rate compression experiments were performed using a compressive split-Hopkinson bar consisting of $25 \mathrm{~mm}$ diameter input and output rods of maraging steel.

High-speed photographs were acquired using a SIMX16 camera, manufactured by Specialised Imaging Ltd, which produces 16 frames of a little over 1 MPixel resolution. The frame rate was c.a. $10^{5}$ frames per second, with an exposure time of $2 \mu \mathrm{s}$. In order to produce three images of the specimen at high rates, two mirrors were used: each frame from the camera then contained three views, spaced at $60^{\circ}$.

Experiments conducted under these conditions will be referred to as high strain rate (HR).

\section{Calculating True Stress and True Strain}

For the quasi-static tensile specimens the force was taken from the load cell and the true strain was calculated using displacement data from digital image correlation (DIC) tracking of the specimen shoulders. For the quasi-static compression specimens the force was again taken from the load cell, with the true strain calculated by DIC of the region where the anvils and specimen meet.

The analysis procedure for the split-Hopkinson pressure bar experiments must take into account the fact that the input bar begins moving before the stress wave reaches the output bar. This means that an apparent strain is calculated before the stress at the output bar interface begins to rise, and some means of correcting for this must be adopted. High-speed photography provides an opportunity to correct the data for this effect and the strain accumulated outside the gauge length of the specimen.

DIC was used to track the motion of the specimen shoulders from the high-speed photographs taken during the high strain rate experiments. 16 measurements were made, one for each frame from the camera, and the correlation was performed by comparison to a series of 16 static images taken before the experiment started. Using the 16 strain values, the strain produced from the interface velocities was then shifted and scaled. The true strain rate experienced in the neck of the specimen was calculated to be $3.1 \times 10^{3} \mathrm{~s}^{-1}$ for the tensile specimens and $3.3 \times 10^{3} \mathrm{~s}^{-1}$ for the compression specimens.

Data obtained from tensile tests are generally limited by the onset of necking. In particular, once necking has occurred, even if the material volume remains constant, true stress cannot be calculated accurately from the applied load and global deformation rate alone. However, using data from the geometry reconstruction technique it is possible to characterise the deforming shape of tensile specimens post-necking, allowing the mean direct true stress to be calculated using the minimum cross-sectional area.

Despite careful specimen design and good lubrication [27], some barrelling was present in all of the compression specimens in this study and therefore the stress was calculated using the maximum cross-sectional area from the geometry reconstruction, rather than assuming the ideal uniform cross-section.

\section{Results and Discussion}

\section{Uniaxial Stress-Strain Behaviour}

Using the methodology outlined above, true stress-strain plots from representative specimens were produced for all the previously outlined experimental conditions. The truestress strain response from RD1, RD2 and TT at quasistatic and high strain rates in both tension and compression are given in Fig. 10a-c, respectively.

From the data presented, a number of clear trends can be seen linking the macroscopic crystallographic texture of the Ti-6Al-4V plate with its mechanical behaviour.

Under all conditions, it is observed that the strength of texturing of the (0001) plane of the $\alpha$ phase in a given material orientation strongly correlates with the observed macroscopic yield/flow stress of the specimens cut from that orientation. 

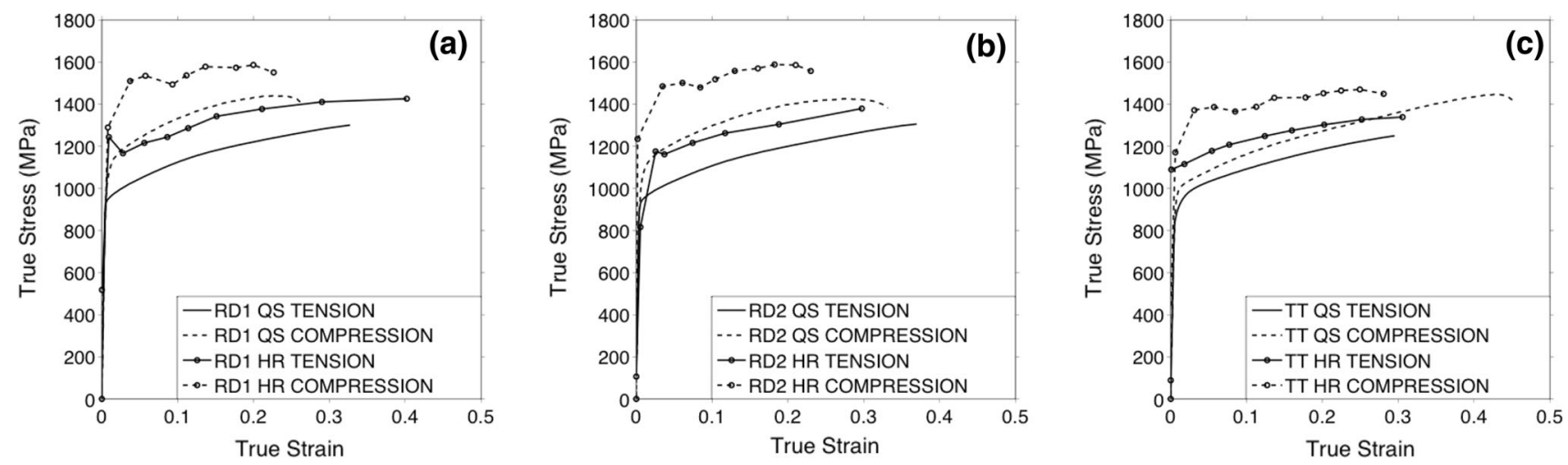

Fig. 10 Representative true stress strain plots at quasi-static (QS) and high strain rates (HR), using maximum (compression) or minimum (tension) cross-sectional area to calculate stress and DIC to measure strain for $\mathbf{a}$ RD1, b RD2 and $\mathbf{c}$ TT
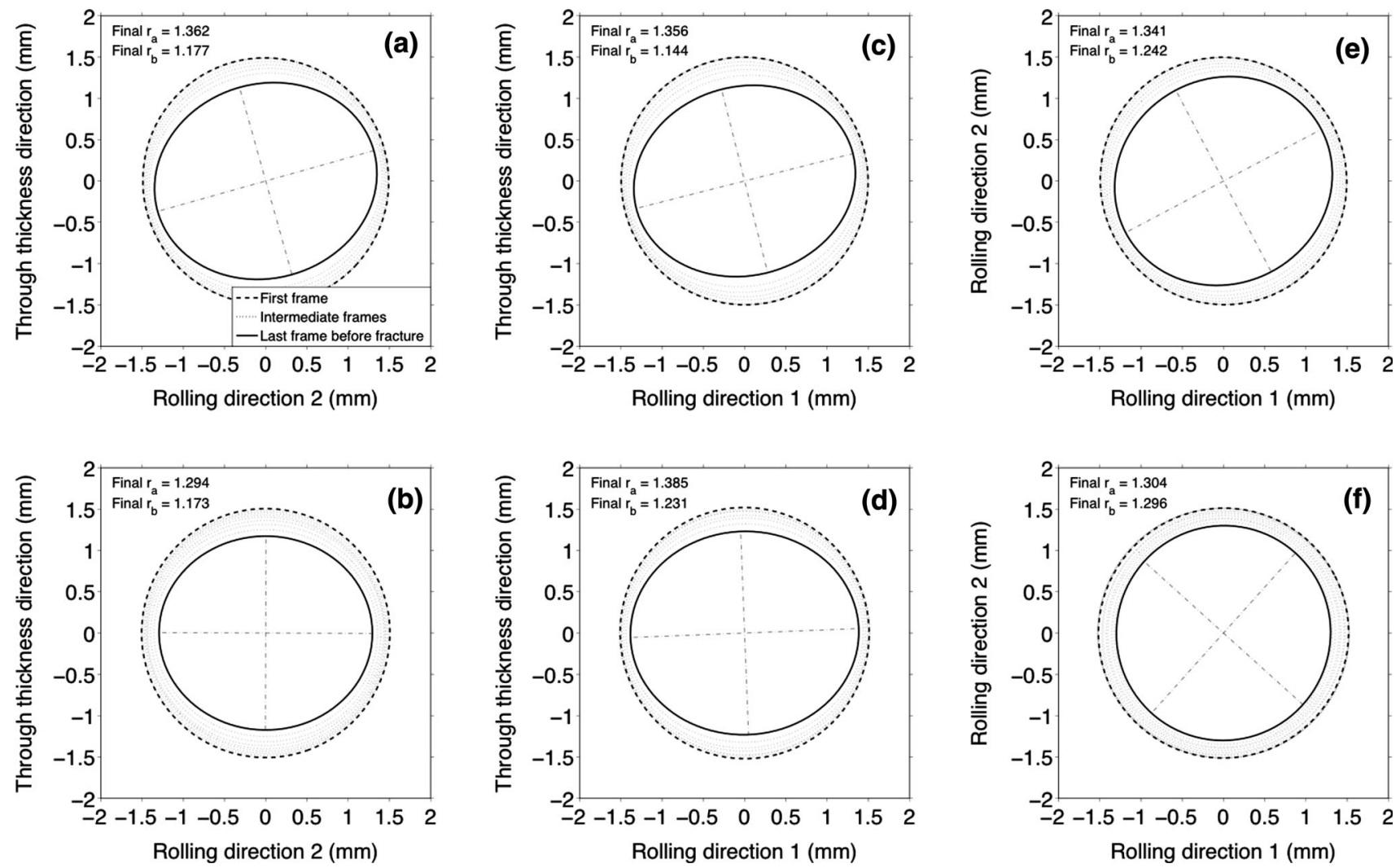

Fig. 11 Evolution of the minimum cross-sectional profile during tension testing in; RD1 at a quasi-static and $\mathbf{b}$ high strain rate, RD2 at $\mathbf{c}$ quasistatic and $\mathbf{d}$ high strain rate and TT at $\mathbf{e}$ quasi-static and $\mathbf{f}$ high strain rate

For example, in Fig. 10, it can be seen that RD1 has the highest yield/flow stress under all conditions, followed by $\mathrm{RD} 2$, then TT. This correlates well with the pole figure for the (0001) plane given in Fig. 5, which shows that RD1 has the highest preferred orientation of (0001) planes, followed by RD2, then TT.

This can be explained qualitatively by considering the relative accessibility to $\langle a\rangle$ type slip systems in the different material orientations. For example, when compared with loading in TT, loading in RD1 or RD2 results in less grains being well aligned for $\langle a\rangle$ type slip. Experiments on $\mathrm{Ti}-\mathrm{Al}$ single crystals by Williams et al. [6] have shown that the ratio of slip system strength between $\langle c+a\rangle$ type slip and $\langle a\rangle$ type slip is of the order of 3-4 at room temperature, although more recent results set the value at 1-1.5 [28]. Therefore, when loading along a given material direction, increasing the number of grains with their c-axis orientated in the direction of loading will result in a higher macroscopic yield strength, as observed here. However, it should be noted that polycrystalline deformation is a complex 
event, with detailed crystal-based models, such as Taylortype models [29], self-consistent models [30] and crystalscale finite element models [31], required to truly quantify effects of preferred crystallographic orientation.

Interestingly, under tensile loading, the effect of the macrotexture on the yield/flow stress behaviour is relatively small. However, under compressive loading, a large difference between the yield/flow stresses in the different material orientations is observed. Further, loading in RD1 and RD2 results in a larger tension/compression asymmetry than loading in TT.

The mechanism responsible for the observed orientation dependent tension/compression asymmetry in titanium and its alloys is currently unclear. Possible mechanisms include the hydrostatic pressure dependence of $\langle c+a\rangle$ slip [32], the tension/compression dependence of deformation twinning [33] and residual stresses locked into the material during thermo-mechanical processing.

\section{Evolving Specimen Cross-Sections}

The evolving minimum (tension) and maximum (compression) cross-sectional profiles from these representative specimens, as well as the major and minor cross-sectional radii of the fitted ellipses, were determined for all the experimental conditions. The minimum cross-sectional profiles during tension testing in RD1, RD2 and TT at quasi-static and high strain rates are given in Fig. 11. The maximum cross-sectional profiles during compression testing in RD1, RD2 and TT at quasi-static and high strain rates are given in Fig. 12.

To quantify the anisotropic evolution of the specimen cross-sections during mechanical loading, data from the fitted ellipses were used to calculate the ratio, $R$, of the major and minor cross-sectional true radial strains:

$R=\frac{\ln \left(\frac{r_{a}}{r_{0}}\right)}{\ln \left(\frac{r_{b}}{r_{0}}\right)}$

where $r_{a}$ is the current major cross-sectional radius, $r_{b}$ is the current minor cross-sectional radius and $r_{O}$ is the initial cross-sectional radius. The ratios were calculated as a function of the true area strain, $\varepsilon_{A}$ :

$\varepsilon_{A}=\ln \left(\frac{A}{A_{0}}\right)$

where $A$ is the current cross-sectional area and $A_{0}$ is the initial cross-sectional area. The results of this analysis for
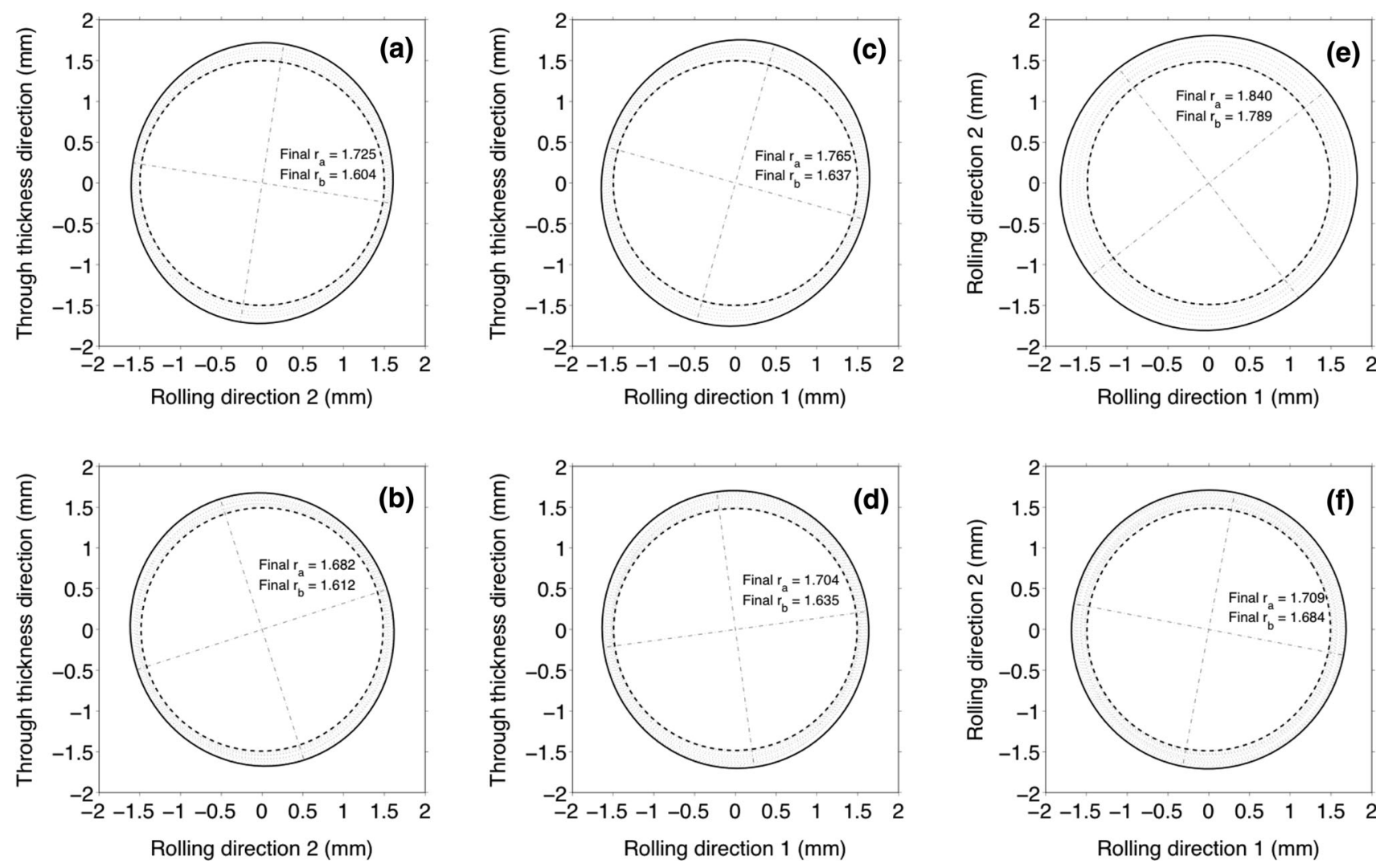

Fig. 12 Evolution of maximum cross-sectional profile during compression testing in; RD1 at a quasi-static and b high strain rate, RD2 at $\mathbf{c}$ quasi-static and $\mathbf{d}$ high strain rate and TT at $\mathbf{e}$ quasi-static and $\mathbf{f}$ high strain rate 
all quasi-static and high strain rate conditions are given in Fig. 13a, b, respectively.

The correlation between macroscopic texture and the uniaxial yield/flow stress behaviour discussed previous is also mirrored in the results from the cross-sectional reconstruction of the plastically deforming specimens. From Figs. 11 and 12, it can be seen that loading in RD1 and RD2 results in an elliptical cross-section being formed in the specimen, whereas loading in TT results in a near circular cross-section being formed. This is clearly due to the varying macroscopic yield/flow stresses in the different material orientations, which in turn is due to the preferred crystallographic orientation of the $\alpha$ phase, as discussed previously. Loading in TT results in a relatively equal distribution of grains with their (0001) planes orientated in the specimen cross-sectional directions, thus a near-circular cross-section is formed during plastic deformation.
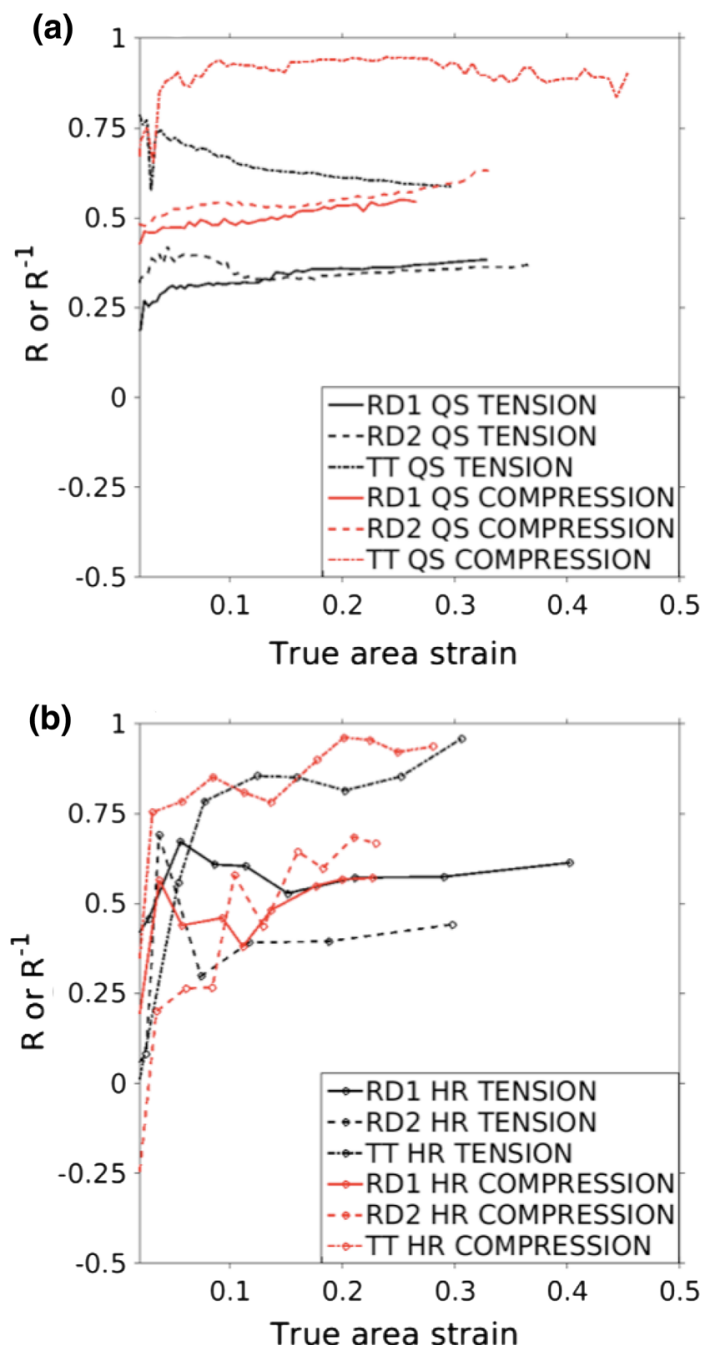

Fig. 13 Evolution in the ratio between the major and minor true radial strain of the deforming specimen cross-sections as a function of true area strain during a quasi-static testing and $\mathbf{b}$ high strain rate testing
However, loading in either RD1 or RD2 results in perpendicular specimen cross-sectional directions with low and high distributions of grains with their (0001) planes orientated in those directions, thus an elliptical cross-section is formed during plastic deformation.

This effect can also be observed in the true radial strain ratio plots shown in Fig. 13, where it can be seen that loading in RD1 and RD2 under all experimental conditions results in a highly anisotropic evolution of the cross-sectional profiles. On the other hand, loading in TT under all conditions results in a significantly less anisotropic evolution of the cross-sectional profiles.

It can also be observed that when loading in RD1 and RD2, the radial strain ratios show good agreement between tension and compression. However, when loading in TT, there is a marked difference in the true radial strain ratios between the tension and compression specimens at quasistatic rates.

\section{Conclusions}

A cross-rolled plate of the industrially important titanium alloy, Ti-6Al-4V, has been microstructurally and mechanically characterised using a range of different experimental techniques. The macroscopic stress-strain response and the evolution of the specimen geometries have been characterised over different experimental conditions and qualitatively linked to the macroscopic crystallographic texture in the plate.

In particular, it has been shown that the strength of texturing of the (0001) plane of the $\alpha$ phase in a given material orientation strongly correlates with the observed macroscopic yield/flow stress of the specimens cut from that orientation. This was qualitatively explained by considering the relative accessibility to $\langle a\rangle$ type slip systems in the different material orientations. Further to this point, a material orientation dependent tension/compression asymmetry in the uniaxial yield/flow stress was observed.

The correlation between macroscopic texture and the uniaxial yield/flow stress behaviour was mirrored in the results from the cross-sectional reconstruction, with specimens producing deformed cross-sectional profiles that were dependent on the orientation of the crystallographic texture relative to the specimen.

It is hoped that these data can be used to inform and validate material models capable of incorporating the influence of macroscopic crystallographic texture on the mechanical behaviour of Ti-6Al-4V.

Acknowledgments The authors would like to thank Prof. Paul Dawson for access to the ODFPF software package, which was used to calculate the orientation distribution function and generate the pole figures. 


\section{References}

1. Lutering G, Williams J (2003) Titanium. Springer, Berlin

2. Donachie M (2000) Titanium: a technical guide. ASM International, Materials Park

3. Leyens C, Peters M (2003) Titanium and titanium alloys. Wiley, Weinheim

4. Froes FH, Friedrich H, Kiese J, Bergoint D (2004) Titanium in the family automobile: the cost challenge. JOM 56:40-44

5. Burgers W (1934) On the process of transition of the cubic-bodycentered modification into the hexagonal-close-packed modification of zirconium. Physica 1:561-586

6. Williams JC, Baggerly RG, Paton NE (2002) Deformation behavior of $\mathrm{HCP}$ Ti-Al alloy single crystals. Metall Mater Trans A 33:837-850

7. Ramesh KT (2002) Effects of high rates of loading on the deformation behavior and failure mechanisms of hexagonal close-packed metals and alloys. Metall Mater Trans A 33:927-935

8. Yoo MH (1981) Slip, twinning, and fracture in hexagonal closepacked metals. MTA 12:409-418

9. Chichili D, Ramesh K, Hemker K (1998) The high-strain-rate response of alpha-titanium: experiments, deformation mechanisms and modeling. Acta Mater 46:1025-1043

10. Salem AA, Kalidindi SR, Doherty RD (2003) Strain hardening of titanium: role of deformation twinning. Acta Mater 51:4225-4237

11. Yapici GG, Karaman I, Luo Z-P (2006) Mechanical twinning and texture evolution in severely deformed Ti-6Al-4V at high temperatures. Acta Mater 54:3755-3771

12. Prakash DGL, Ding R, Moat R, Jones I, Withers P, da Fonseca JQ et al (2010) Deformation twinning in Ti-6Al-4V during low strain rate deformation to moderate strains at room temperature. Mater Sci Eng A 527:5734-5744

13. Wielewski E, Appleby-Thomas G, Hazell P, Hameed A (2013) An experimental investigation into the micro-mechanics of spall initiation and propagation in Ti-6Al-4V during shock loading. Mater Sci Eng A 578:331-339

14. Dunne FPE, Rugg D (2008) On the mechanisms of fatigue facet nucleation in titanium alloys. Fatigue Fract Eng Mater Struct 31:949-958

15. Bernier J, Park J-S, Pilchak A, Glavicic M, Miller M (2008) Measuring stress distributions in Ti-6Al-4V using synchrotron X-ray diffraction. Metall Mater Trans A 39:3120-3133

16. Kumar A, Dawson PR (1998) Modeling crystallographic texture evolution with finite elements over neo-Eulerian orientation spaces. Comput Methods Appl Mech Eng 153:259-302

17. Bantounas I, Lindley TC, Rugg D, Dye D (2007) Effect of microtexture on fatigue cracking in Ti-6Al-4V. Acta Mater 55:5655-5665

18. Bridier F, Villechaise P, Mendez J (2008) Slip and fatigue crack formation processes in an $\alpha / \beta$ titanium alloy in relation to crystallographic texture on different scales. Acta Mater 56:3951-3962

19. Wielewski E, Siviour CR, Petrinic N (2012) On the correlation between macrozones and twinning in Ti-6Al-4V at very high strain rates. Scr Mater 67:229-232

20. Arthington MR, Siviour CR, Petrinic N, Elliott BCF (2009) Cross-section reconstruction during uniaxial loading. Meas Sci Technol 20:075701

21. Arthington M, Siviour C, Petrinic N (2012) Improved materials characterisation through the application of geometry reconstruction to quasi-static and high-strain-rate tension tests. Int J Impact Eng 46:86-96

22. Wielewski E, Arthington MR, Siviour CR, Carter S, Hofmann F, Korsunsky AM et al (2010) A method for the in situ measurement of evolving elliptical cross-sections in initially cylindrical Taylor impact specimens. J Strain Anal Eng Des 45:429-437

23. Gray G (2000) Classic split-hopkinson pressure bar testing. ASM International, Materials Park

24. Field J, Walley S, Proud W, Goldrein H, Siviour C (2004) Review of experimental techniques for high rate deformation and shock studies-Fifth International Symposium on Impact Engineering. Int J Impact Eng 30:725-775

25. Harding J, Wood EO, Campbell JD (1960) Tensile testing of materials at impact rates of strain. Proc Inst Mech Eng Part C 2:88-96

26. Harding J, Welsh LM (1983) A tensile testing technique for fibrereinforced composites at impact rates of strain. J Mater Sci 18:1810-1826

27. Li P, Siviour CR, Petrinic N (2009) The effect of strain rate, specimen geometry and lubrication on responses of aluminium AA2024 in uniaxial compression experiments. Exp Mech 49:587-593

28. Ding R, Gong J, Wilkinson AJ, Jones IP (2014) $\langle\mathrm{c}+\mathrm{a}\rangle$ dislocations in deformed Ti-6Al-4V micro-cantilevers. Acta Mater $76: 127-134$

29. Taylor G (1938) Plastic strain in metals. J Inst Met 62:307-324

30. Lebensohn R, Tomé C (1993) A self-consistent anisotropic approach for the simulation of plastic deformation and texture development of polycrystals: application to zirconium alloys. Acta Metall Mater 41:2611-2624

31. Marin E, Dawson P (1998) Elastoplastic finite element analyses of metal deformations using polycrystal constitutive models. Comput Methods Appl Mech Eng 165:23-41

32. Jones I, Hutchinson W (1981) Stress-state dependence of slip in Titanium-6Al-4V and other H.C.P. metals. Acta Metall 29:951-968

33. Nixon ME, Cazacu O, Lebensohn RA (2010) Anisotropic response of high-purity $\alpha$-titanium: experimental characterization and constitutive modeling. Int J Plast 26:516-532 